\title{
On the Effective Rate and Energy Detection Based Spectrum Sensing over $\alpha-\eta-\kappa-\mu$ Fading Channels
}

\author{
Hussien Al-Hmood, Member, IEEE, and H. S. Al-Raweshidy, Senior Member, IEEE
}

\begin{abstract}
In this paper, the effective rate (ER) of wireless communication systems and the performance of energy detection (ED) based spectrum sensing over $\alpha-\eta-\kappa-\mu$ fading channels are analysed. To this end, novel mathematically tractable exact expressions of the ER, the average detection probability (ADP) and the average area under the receiver characteristics curve (AUC) are derived. The asymptotic expressions at high average signal-to-noise ratio (SNR) values are also provided. A comparison between the numerical results and Monte Carlo simulations is presented to verify the validation of our analysis. The mathematical relationship between the ER and the performance metrics of the ED which is based on the time-bandwidth product, is explained. The provided results show that the increase in the time-bandwidth product from 1 to 4 reduces the ER, the ADP, and the average AUC by nearly $21 \%, 8 \%$, and $3 \%$, respectively, for constant average SNR at $15 \mathrm{~dB}$ and delay exponent at 0.1 .
\end{abstract}

Index Terms-effective rate, energy detection, $\alpha-\eta-\kappa-\mu$ fading channel.

\section{INTRODUCTION}

$\mathbf{T}$ O model the multipath fading impacts of wireless channels, different distributions have been used in the open literature such as Nakagami- $m$ and Nakagami- $n$ [1]. However, in [2]-[3], the authors explained that the results of these distributions don't match well with the field measurements. This is because the derivation of these distributions is based on an assumption of a homogeneous diffuse scattering field. Consequently, several generalised distributions have been suggested to provide better fitting to the practical data via considering the multipath waves of the clusters of the transmitted signal are propagated in non-homogeneous and/or non-linear environments (see [2]-[5] and references therein). Moreover, these distributions comprise most of the well-known classic fading conditions as special cases. For instance, the $\kappa-\mu$ and the $\eta-\mu$ fading channels are employed to represent the line-of-sight (LoS) and the non-LoS (NLoS) fading conditions, respectively [2]. In addition, the $\alpha-\mu$ fading is used for the non-linear environment of communication systems [3]. In [4], the $\alpha-\eta-\mu$ fading channel is presented as a unified framework for $\alpha-\mu$ and $\eta-\mu$ as well as the $\alpha-\kappa-\mu$ fading is proposed as a combined model for both $\alpha-\mu$ and $\kappa-\mu$ distributions.

Recently, the authors in [5] proposed the so-called $\alpha-\eta-$ $\kappa-\mu$ fading channels as a unified model for $\alpha-\eta-\mu$ and

Copyright (c) 2015 IEEE. Personal use of this material is permitted. However, permission to use this material for any other purposes must be obtained from the IEEE by sending a request to pubs-permissions@ieee.org.

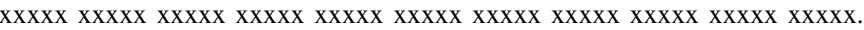

Hussien Al-Hmood is with the Department of Electrical and Electronics Engineering, College of Engineering, University of Thi-Qar, Iraq, e-mails: (Hussien.Al-Hmood@brunel.ac.uk; H.A.Al-Hmood@ieee.org).

H. S. Al-Raweshidy is with the Department of Electronic and Computer Engineering, College of Engineering, Design and Physical Sciences, Brunel University London, UK, e-mail: Hamed.Al-Raweshidy @brunel.ac.uk. $\alpha-\kappa-\mu$ distributions. Hence, this representation includes nearly all the generalised LoS, NLoS, and non-linear fading scenarios. Accordingly, the $\alpha-\eta-\kappa-\mu$ fading has been used to analyse the channel capacity under different transmission policies [6] and the physical layer security [7]. In [8], the outage and error probabilities as well as the channel capacity are given.

In this paper, the effective rate (ER) and the energy detection (ED) based spectrum sensing (SS) over $\alpha-\eta-\kappa-\mu$ fading channels that have not been yet studied by previous works, are analysed. Additionally, the relationship between the ER and the performance of ED is also explained.

Our main contributions are summarized as follows:

- We derive novel exact mathematically tractable expression of the ER of $\alpha-\eta-\kappa-\mu$ fading channels.

- The performance of ED based SS over $\alpha-\eta-\kappa-\mu$ fading channels is analysed via deriving the average detection probability (ADP) and the average area under the receiver operating characteristics curve (AUC). To this end and to the best of the authors' knowledge, novel mathematically tractable expressions are obtained.

- We derive the asymptotic expressions for the ER and average AUC to gain further insights into the impacts of the fading parameters on the system performance.

- We employ a highly accurate method to compute the number of the truncated terms that makes the infinite series of the probability density function (PDF) converges steadily and quickly for a certain figure of accuracy.

- Unlike [9] in which the relationship between the entropy and the ED over $\mathcal{F}$ fading channel is explained via studying the impact of the channel parameters on them, we prove that the ER and the performance metrics of the ED are mathematically related by the time-bandwidth product. This relationship gives good insights on how the ED of the signal and the quality of service (QoS) are simultaneously influenced by the time-bandwidth product.

\section{THE $\alpha-\eta-\kappa-\mu$ FADING CHANNEL MODEL}

The signal envelope, $R$, in $\alpha-\eta-\kappa-\mu$ fading channel can be expressed as [5, eq. (9)]

$$
R^{\alpha}=\sum_{i=1}^{\mu_{x}}\left(X_{i}+\lambda_{x_{i}}\right)^{2}+\sum_{i=1}^{\mu_{y}}\left(Y_{i}+\lambda_{y_{i}}\right)^{2}
$$

where $X_{i}$ and $Y_{i}$ are mutually independent Gaussian processes with $\mathbb{E}\left[X_{i}\right]=\mathbb{E}\left[Y_{i}\right]=0, \mathbb{E}\left[X_{i}\right]=\sigma_{x}^{2}$ and $\mathbb{E}\left[X_{i}\right]=\sigma_{y}^{2}$, with $\mathbb{E}[$.$] denoting statistical expectation, while \lambda_{x_{i}}$ and $\lambda_{y_{i}}$ are, respectively, the mean values of the in-phase and quadrature components of the multipath waves of the cluster $i$. Moreover, 
$\alpha$ represents the non-linearity parameter while $\mu_{x}$ and $\mu_{y}$ stand for the number of multipath clusters of in-phase and quadrature components, respectively. The mean value of $R^{\alpha}$ is given by $\hat{r}^{\alpha}=\mathbb{E}\left[R^{\alpha}\right]=\mu_{x} \sigma_{x}^{2}+\lambda_{x}^{2}+\mu_{x} \sigma_{y}^{2}+\lambda_{y}^{2}$ where $\lambda_{x}^{2}=\sum_{i=1}^{\mu_{x}} \lambda_{x_{i}}^{2}$ and $\lambda_{y}^{2}=\sum_{i=1}^{\mu_{y}} \lambda_{y_{i}}^{2}$. Hence, the average signal-to-noise (SNR), $\bar{\gamma}$, is expressed as $\bar{\gamma}=\mathbb{E}[\gamma]=\hat{r}^{\alpha} E_{s} / N_{0}$, where $\gamma$ is the instantaneous SNR, $E_{s}$ is the energy per symbol, and $N_{0}$ is the one-sided power spectral density of the additive white Gaussian noise (AWGN).

According to [5], the $\alpha-\eta-\kappa-\mu$ fading model can be described by using the following non-zero positive parameters:

i) $\eta=\frac{\mu_{x} \sigma_{x}^{2}}{\mu_{y} \sigma_{y}^{2}}$ is the ratio of the total powers of the in-phase and quadrature scattered waves of the multipath cluster.

ii) $\kappa=\frac{\lambda_{x}^{2}+\lambda_{y}^{2}}{\mu_{x} \sigma_{x}^{2}+\mu_{y} \sigma_{y}^{2}}$ represents the ratio between the total powers of the dominant components and scattered waves.

iii) $\mu=\frac{\mu_{x}+\mu_{y}}{2}$ denotes the real extension of the total number of multipath clusters.

iv) $p=\frac{\mu_{x}}{\mu_{y}}$ stands for the ratio of the number of the multipath clusters of in-phase and quadrature signals.

v) $q=\frac{\lambda_{x}^{2} \mu_{y} \sigma_{y}^{2}}{\lambda_{y}^{2} \mu_{x} \sigma_{x}^{2}}$ is defined as the ratio of two ratios which are the ratio of the power of the dominant components to the power of the scattered waves of the in-phase signal and its counterpart ratio for the quadrature signal.

The PDF of $\gamma$ over $\alpha-\eta-\kappa-\mu$ fading channels can be written as [5, eq. (29)]

$$
f_{\gamma}(\gamma)=\sum_{l=0}^{\infty} \sum_{j=0}^{l} \frac{\alpha(-1)^{j} 2^{j-\mu-1} l ! c_{l}}{\Gamma(\mu+j)(l-j) ! j ! \bar{\gamma}^{\varphi}} \gamma^{\varphi-1} e^{-\frac{\gamma^{\frac{\alpha}{2}}}{2 \bar{\gamma}^{\frac{\alpha}{2}}}}
$$

where $\varphi=\frac{\alpha(\mu+j)}{2}, \Gamma(a)=\int_{0}^{\infty} x^{a-1} e^{-x} d x$ is the incomplete Gamma function [10, eq. (8.310.1)] and $c_{l}$ can be recursively calculated by $[5$, eq. (15)]

$$
c_{l}=\frac{1}{l} \sum_{r=0}^{l-1} c_{r} d_{l-r} \quad l \geqslant 1
$$

where $d_{l-r}$ can be computed by [5, eq. (31)]

$$
\begin{aligned}
d_{j} & =\frac{\mu}{1+p}\left(\frac{2-\psi}{2+3 \psi}\right)^{j}-\frac{8 j \kappa \eta p^{2} q(2 p-\psi \eta)^{j-1} \bar{\gamma}^{\alpha}}{(1+\eta q)(1+\kappa)(2 p+3 \psi \eta)^{j+1}} \\
& +\frac{\mu p}{1+p}\left(\frac{2 p-\psi \eta}{2 p+3 \psi \eta}\right)^{j}-\frac{8 j \kappa(2 p-\psi)^{j-1} \bar{\gamma}^{\alpha}}{(1+\eta q)(1+\kappa)(2 p+3 \psi \eta)^{j+1}}
\end{aligned}
$$

with $\psi=\frac{(1+p) \bar{\gamma}^{\alpha}}{\mu(1+\eta)(1+\kappa)}$ and the initial value of $c_{l}$, i.e. $c_{0}$, can be evaluated by [5, eq. (30)]

$$
c_{0}=\frac{8^{\mu}(2+3 \psi)^{-\frac{\mu}{1+p}}\left(2+3 \psi \frac{\eta}{p}\right)^{-\frac{\mu p}{1+p}}}{\exp \left(\frac{3 \kappa(2 p(1+\eta q)+3 \psi \eta(1+p q))}{(1+\eta q)(1+\kappa)(2+3 \psi)(2+3 \psi \eta)} \bar{\gamma}^{\alpha}\right)}
$$

Using the identity [11, eq. (1.39)], (2) can be rewritten as

$$
f_{\gamma}(\gamma)=\sum_{l=0}^{\infty} \sum_{j=0}^{l} \frac{\alpha(-1)^{j} 2^{j-\mu-1} l ! c_{l} \gamma^{\varphi-1}}{\Gamma(\mu+j)(l-j) ! j ! \bar{\gamma}^{\varphi}} H_{0,1}^{1,0}\left[\frac{\gamma^{\frac{\alpha}{2}}}{2 \bar{\gamma}^{\frac{\alpha}{2}}} \mid \begin{array}{c}
- \\
(0,1)
\end{array}\right]
$$

where $H_{u, v}^{s, t}[$.$] is the Fox's H$-function (FHF) defined in [11, eq. (1.2)]
When $\bar{\gamma} \rightarrow \infty$, the exponential function in (2) tends to unity. Thus, the asymptotic of the PDF of $\gamma, f_{\gamma}^{\text {Asy }}(\gamma)$ is deduced as

$$
f_{\gamma}^{\text {Asy }}(\gamma) \approx \sum_{l=0}^{\infty} \sum_{j=0}^{l} \frac{\alpha(-1)^{j} 2^{j-\mu-1} l ! c_{l}}{\Gamma(\mu+j)(l-j) ! j ! \bar{\gamma}^{\varphi}} \gamma^{\varphi-1}
$$

\section{Performance of $\alpha-\eta-\kappa-\mu$ Fading Channels}

\section{A. Effective Rate}

The ER has been proposed to measure the performance of the wireless communication systems under the QoS constraints, such as system delays, that have not been taken into consideration by Shannon [1].

The ER can be calculated by [12, eq. (4)]

$$
\mathrm{R}=-\frac{1}{\mathrm{~A}} \log _{2}\left(\int_{0}^{\infty}(1+\gamma)^{-\mathrm{A}} f_{\gamma}(\gamma) d \gamma\right)
$$

where $\mathrm{A} \triangleq \theta u / \ln 2$ with $\theta$ denotes the delay exponent and $u=T B$ is the product of the time $(T)$ and bandwidth $(B)$.

Substituting (6) into (8) and using the property [11, eq. (1.126)] to express $(1+\gamma)^{-\mathrm{A}}$ in terms of the FHF, the following integral is yielded

$$
\int_{0}^{\infty} \gamma^{\varphi-1} H_{1,1}^{1,1}\left[\gamma \mid \begin{array}{c}
(1-\mathrm{A}, 1) \\
(0,1)
\end{array}\right] H_{0,1}^{1,0}\left[\frac{\gamma^{\frac{\alpha}{2}}}{2 \bar{\gamma}^{\frac{\alpha}{2}}} \mid \begin{array}{c}
- \\
(0,1)
\end{array}\right] d \gamma
$$

Making use of [11, pp. 60] for (9) and inserting the result in (8) along with the remaining terms of (6), the ER is derived in exact expression as shown in (10) at the top of the next page.

For the asymptotic behaviour of the ER, $\mathrm{R}^{\text {Asy }}$, we substitute (7) into (8). Hence, the following integral is the result

$$
\int_{0}^{\infty}(1+\gamma)^{-\mathrm{A}} \gamma^{\varphi-1} d \gamma \stackrel{\left(a_{1}\right)}{=} \frac{\mathrm{B}(\varphi, \mathrm{A}-\varphi)}{\Gamma(\mathrm{A})}
$$

where $\left(a_{1}\right)$ follows [10, eq. (3.194.3)] and $\mathrm{B}(a, b)$ is the incomplete Beta function [10, eq. (8.384.1)].

Inserting the result of (11) and the terms of (7) that have not been used in the integral of (11), in (8), we have $\mathrm{R}^{\text {Asy }}$ as

$$
\mathrm{R}^{\mathrm{Asy}} \approx-\frac{1}{\mathrm{~A}} \log _{2}\left(\sum_{l=0}^{\infty} \sum_{j=0}^{l} \frac{\alpha(-1)^{j} 2^{j-\mu-1} l ! c_{l} \mathrm{~B}(\varphi, \mathrm{A}-\varphi)}{\Gamma(\mathrm{A}) \Gamma(\mu+j)(l-j) ! j ! \bar{\gamma}^{\varphi}}\right)
$$

\section{B. Energy Detection Based Spectrum Sensing}

At the output of ED, the received signal, $r(t)$, is given as [13]

$$
r(t)= \begin{cases}w(t) & : \mathrm{H}_{0} \\ h(t) s(t)+w(t) & : \mathrm{H}_{1}\end{cases}
$$

where $s(t)$ is the transmitted signal, $h(t)$ is the channel gain and $w(t)$ represents the noise which is assumed to be AWGN. Additionally, in (13), it can be noted there are two binary hypothesis, namely, $\mathrm{H}_{0}$ and $\mathrm{H}_{1}$. The former means that $s(t)$ is absent whereas the latter signifies $s(t)$ is present. These hypothesis are deuced via comparing a predetermined threshold value, $\lambda$, with the test statistic, $\Delta$, which is computed 


$$
\mathrm{R}=-\frac{1}{\mathrm{~A}} \log _{2}\left(\sum_{l=0}^{\infty} \sum_{j=0}^{l} \frac{\alpha(-1)^{j} 2^{j-\mu-1} l ! c_{l}}{\Gamma(\mathrm{A}) \Gamma(\mu+j)(l-j) ! j ! \bar{\gamma} \varphi} H_{1,2}^{2,1}\left[2^{\frac{2}{\alpha}} \mid \begin{array}{c}
\left(1-\varphi, \frac{\alpha}{2}\right) \\
(0,1),\left(\mathrm{A}-\varphi, \frac{\alpha}{2}\right)
\end{array}\right]\right)
$$

$$
\begin{aligned}
& \bar{P}_{d}=1-\pi \lambda^{u} \sum_{l=0}^{\infty} \sum_{j=0}^{l} \frac{\alpha(-1)^{j} 2^{j-\mu-u-1} l ! c_{l}}{\Gamma(\mu+j)(l-j) ! j ! \bar{\gamma}^{\varphi}} \\
& \times H_{2,1: 0,1 ; 1,3 ; 0,1}^{0,2: 1,0 ; 1,0 ; 1,0}\left[\frac{\lambda}{2}, \frac{\lambda}{2}, \frac{1}{2 \bar{\gamma}^{\frac{\alpha}{2}}}\left|\begin{array}{c}
(1-u ; 1,1,0) ;\left(1-\varphi ; 0,1, \frac{\alpha}{2}\right) \\
(-u ; 1,1,0)
\end{array}\right| \begin{array}{c}
- \\
(0,1)
\end{array}|(0,1),(1-u, 1),(0.5,1)|(0,1)\right]
\end{aligned}
$$

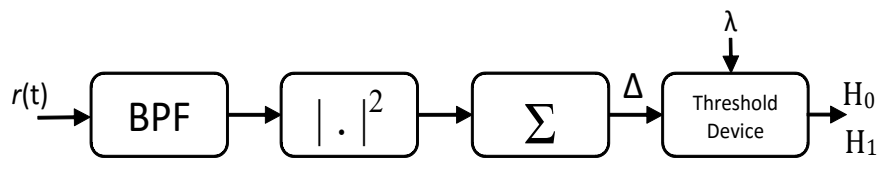

Fig. 1. System model of energy detection [9].

after filtering $r(t)$ by a band-pass filter (BPF) with $B$ and then using square and sum devices over $T$ as shown in Fig. 1.

Based on the comparison above, the performance of ED can be analysed by the detection probability, $P_{d}$, which is present when $\Delta>\lambda$ and $\mathrm{H}_{1}$ are true. The second metric is the false alarm probability, $P_{f a}$, which is obtained when both $\Delta>\lambda$ and $\mathrm{H}_{0}$ are true. It is worth mentioning that the detectability of ED enhances when the $P_{d}$ is high and $P_{f a}$ is low. This is because a large $P_{d}$ means there is no harmful interference between the licensed and unlicensed users whereas the small value of $P_{f a}$ leads to an efficient usage of the spectrum band.

When there is no fading effect, i.e., under AWGN channel, the $P_{d}$ and $P_{f a}$ are expressed as [13, eq. (5) /eq. (4)]

$$
P_{d}=\operatorname{Pr}\left\{\Delta>\lambda \mid \mathrm{H}_{1}\right\}=Q_{u}(\sqrt{2 \gamma}, \sqrt{\lambda})
$$

and

$$
P_{f a}=\operatorname{Pr}\left\{\Delta>\lambda \mid \mathrm{H}_{0}\right\}=\frac{\Gamma\left(u, \frac{\lambda}{2}\right)}{\Gamma(u)}
$$

where $\operatorname{Pr}\{$.$\} denotes the probability, Q_{x}(a, b)$ is the generalized Marcum $Q$-function defined in [1, eq. (4.60)], and $\Gamma(a, b)$ is the upper incomplete Gamma function [9, eq. (8.350.2)].

1) Average Detection Probability: When the distribution of $h(t)$ in (13) is an $\alpha-\eta-\kappa-\mu$ fading, the average of $P_{d}$ over (6) is computed to obtain the ADP, $\bar{P}_{d}$, as [14, eq. (19)]

$$
\bar{P}_{d}=\int_{0}^{\infty} P_{d} f_{\gamma}(\gamma) d \gamma
$$

With the help of $\left[14\right.$, eq. $\left.\left(20 .\left(c_{3}\right)\right)\right]$, the $P_{d}$, i.e., $Q_{u}(\sqrt{2 \gamma}, \sqrt{\lambda})$, of (14) can be represented as

$$
\begin{gathered}
P_{d}=1-\pi e^{-\gamma}\left(\frac{\lambda}{2}\right)^{u} \frac{1}{(2 \pi i)^{2}} \int_{\mathbb{C}_{1}} \int_{\mathbb{C}_{2}} \frac{\Gamma\left(u-t_{1}-t_{2}\right)}{\Gamma\left(1+u-t_{1}-t_{2}\right)} \\
\frac{\Gamma\left(t_{1}\right) \Gamma\left(t_{2}\right)}{\Gamma\left(0.5+t_{2}\right) \Gamma\left(0.5-t_{2}\right) \Gamma\left(u-t_{2}\right)}\left(\frac{\lambda}{2}\right)^{-t_{1}}\left(\frac{\lambda \gamma}{2}\right)^{-t_{2}} d t_{1} d t_{2}
\end{gathered}
$$

where $i=\sqrt{-1}$ and $\mathbb{C}_{k}$ with $k \in\{1,2\}$ is the suitable Barnestype closed contour in the complex $t_{k}$-plane.
Plugging (6) and (17) in (16) and recalling the fact that $\int_{0}^{\infty} f_{\gamma}(\gamma) d \gamma \triangleq 1$, this yields

$$
\begin{aligned}
\int_{0}^{\infty} \gamma^{\varphi-t_{2}-1} e^{-\gamma} H_{0,1}^{1,0}\left[\frac{\gamma^{\frac{\alpha}{2}}}{2 \bar{\gamma}^{\frac{\alpha}{2}}} \mid(0,1)\right] d \gamma \stackrel{\left(a_{2}\right)}{=} \\
H_{1,1}^{1,1}\left[\frac{1}{2 \bar{\gamma}^{\frac{\alpha}{2}}} \mid \begin{array}{c}
\left(1-\varphi+t_{2}, \frac{\alpha}{2}\right) \\
(0,1)
\end{array}\right]
\end{aligned}
$$

where $\left(a_{2}\right)$ arises after recalling [11, eq. (2.19)].

Invoking [11, eq. (1.2)] for the FHF of (18) and then using [11, eq. (A.1)], we obtain (19) as shown at the top of this page. In (19), $H_{u, v: u_{1}, v_{1} ; u_{2}, v_{2} ; u_{3}, v_{3}}^{s, t s_{1}, t_{1} s_{2}, t_{2} ; s_{3}, t_{3}}$ represents the extended generalised bivariate FHF (EGBFHF) defined in [11, eq. (A.1)]. Since the EGBFHF is not yet performed in the popular software packages such as MATLAB and MATHEMATICA, the programming code of [15] is employed to calculate this function.

2) Average AUC: The AUC is a single figure of merit that is proposed as an alternative performance metric to the receiver operating characteristic (ROC) curve which plots the $\bar{P}_{d}$ versus $P_{f a}$. This is because, in sometimes, when two systems are compared using the ROC curve, the intersection between both curves may happen in a certain value of the $P_{f a}$. Accordingly, the result about the superiority of each one on the other is ambiguous [9]. Therefore, the AUC can provide which scheme has better detection capability than the other at different values of $\bar{\gamma}$ and $u$.

For AWGN, the AUC, $\mathcal{A}(\gamma)$, is given as [14, eq. (24)]

$$
\mathcal{A}(\gamma)=1-\sum_{r=0}^{u-1} \sum_{n=0}^{r}\left(\begin{array}{c}
r+u-1 \\
r-n
\end{array}\right) \frac{2^{-(r+n+u)}}{n !} \gamma^{n} e^{-\frac{\gamma}{2}}
$$

The average AUC, $\bar{A}$ can be computed by [9, eq. (36)]

$$
\overline{\mathcal{A}}=\int_{0}^{\infty} \mathcal{A}(\gamma) f_{\gamma}(\gamma) d \gamma
$$

Plugging (6) and (20) in (21) and making utilise of $\int_{0}^{\infty} f_{\gamma}(\gamma) d \gamma \triangleq 1$, we have this integral

$$
\begin{array}{r}
\int_{0}^{\infty} \gamma^{\varphi+n-1} e^{-\frac{\gamma}{2}} H_{0,1}^{1,0}\left[\frac{\gamma^{\frac{\alpha}{2}}}{2 \bar{\gamma}^{\frac{\alpha}{2}}} \mid(0,1)\right] d \gamma \stackrel{\left(a_{3}\right)}{=} \\
2^{\varphi+n} H_{1,1}^{1,1}\left[\frac{2^{\frac{\alpha}{2}-1}}{\bar{\gamma}^{\frac{\alpha}{2}}} \mid \begin{array}{c}
\left(1-\varphi-n, \frac{\alpha}{2}\right) \\
(0,1)
\end{array}\right]
\end{array}
$$

where $\left(a_{3}\right)$ obtains after using [11, eq. (2.19)].

Plugging the result of (22) and the terms of (6) and (20) that have not been inserted in (22), $\overline{\mathcal{A}}$ over $\alpha-\eta-\kappa-\mu$ fading is deduced as given in (23) at the top of the next page. 


$$
\overline{\mathcal{A}}=1-\sum_{l=0}^{\infty} \sum_{j=0}^{l} \frac{\alpha(-1)^{j} l ! c_{l} 2^{j+\varphi-\mu-u-1}}{\Gamma(\mu+j)(l-j) ! j ! \bar{\gamma}^{\varphi}} \sum_{r=0}^{u-1} \sum_{n=0}^{r}\left(\begin{array}{c}
r+u-1 \\
r-n
\end{array}\right) \frac{1}{2^{r} n !} H_{1,1}^{1,1}\left[\frac{2^{\frac{\alpha}{2}}}{2 \bar{\gamma}^{\frac{\alpha}{2}}} \mid \begin{array}{c}
\left(1-n-\varphi, \frac{\alpha}{2}\right) \\
(0,1)
\end{array}\right]
$$

For the asymptotic analysis of $\overline{\mathcal{A}}$, i.e., $\overline{\mathcal{A}}^{\text {Asy }}$, we substitute (7) and (20) into (21) and solve the integral with the help of [10, eq. (3.381.4)]. Accordingly, we obtain

$$
\begin{aligned}
\overline{\mathcal{A}}^{\text {Asy }} \approx 1-\sum_{l=0}^{\infty} \sum_{j=0}^{l} & \frac{\alpha(-1)^{j} l ! c_{l}}{\Gamma(\mu+j)(l-j) ! j ! \bar{\gamma}^{\varphi}} \\
& \sum_{r=0}^{u-1} \sum_{n=0}^{r}\left(\begin{array}{c}
r+u-1 \\
r-n
\end{array}\right) \frac{\Gamma(\varphi+n)}{2^{r} n !}
\end{aligned}
$$

\section{TRuncating OF the PDF OF $\alpha-\eta-\kappa-\mu$ FAding}

One can see that the PDF of $\alpha-\kappa-\eta-\mu$ fading conditions in (2) is included an infinite series. Therefore, a truncating error should be applied to find the number of terms, $N$, that is required to satisfy a specific figure of accuracy, $\epsilon(N)$. In this work, we have employed [16, eq. (5)]

$$
\epsilon(N)=\int_{0}^{\infty} f_{\gamma}(\gamma) d \gamma-\int_{0}^{\infty} \hat{f}_{\gamma}(\gamma) d \gamma
$$

where $\hat{f}_{\gamma}(\gamma)$ is the truncating PDF in (6) for $N$ terms that is expressed as

$$
\hat{f}_{\gamma}(\gamma)=\sum_{l=0}^{N} \sum_{j=0}^{l} \frac{\alpha(-1)^{j} 2^{j-\mu-1} l ! c_{l} \gamma^{\varphi-1}}{\Gamma(\mu+j)(l-j) ! j ! \bar{\gamma}^{\varphi}} H_{0,1}^{1,0}\left[\frac{\gamma^{\frac{\alpha}{2}}}{2 \bar{\gamma}^{\frac{\alpha}{2}}} \mid \begin{array}{c}
- \\
(0,1)
\end{array}\right]
$$

Substituting (6) and (26) into (25) and utilising the fact that $\int_{0}^{\infty} f_{\gamma}(\gamma) d \gamma \triangleq 1$, we have

$$
\begin{array}{r}
\epsilon(N)=1-\frac{\alpha}{2^{\mu+1} \Gamma(\mu)} \sum_{l=0}^{N} \sum_{j=0}^{l} \frac{(-2)^{j} l ! c_{l}}{(\mu)_{j}(l-j) ! j ! \bar{\gamma}^{\frac{\alpha(\mu+j)}{2}}} \\
\int_{0}^{\infty} \gamma^{\frac{\alpha(\mu+j)}{2}-1} H_{0,1}^{1,0}\left[\frac{\gamma^{\frac{\alpha}{2}}}{2 \bar{\gamma}^{\frac{\alpha}{2}}} \mid(0,1)\right] d \gamma
\end{array}
$$

Recalling [11, eq. (2.8)] to calculate the integral in (27) and performing some mathematical simplifications to yield

$$
\epsilon(N)=1-\sum_{l=0}^{N} \sum_{j=0}^{l} \frac{\alpha(-1)^{j} l l_{c_{l}} \bar{\gamma}^{\varphi} \Gamma(\varphi)}{2^{1-j\left(\frac{\alpha}{2}+1\right)} \Gamma(\mu+j)(l-j) ! j !}
$$

\section{Analytical and Simulation Results}

In this section, the numerical and the asymptotic results of our derived expressions are compared with the Monte Carlo simulations that are generated by $10^{6}$ iterations. In all results, $p=q=1$, the minimum $N$ that satisfies $\epsilon(N) \leq 10^{-6}$ is selected to be 40 and $\lambda$ of the ADP is obtained form (15).

Fig. 2 shows the ER for $A=0.75$ whereas Fig. 3 illustrates the average missed detection probability (AMDP), $\bar{P}_{m d}=1-\bar{P}_{d}$, for $\left(P_{f a}, u\right)=(0.1,2)$, and the average complementary of AUC (CAUC) which is $1-\overline{\mathcal{A}}$ for $u=2$. From Fig. 2, it is clear that the ER becomes better when $\bar{\gamma}$ increases at constant $B$. This refers to the increasing in

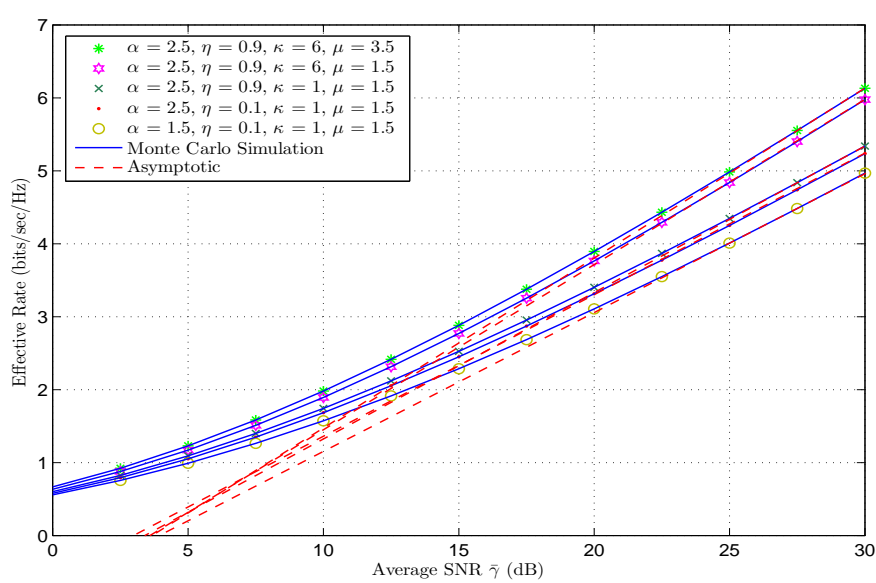

Fig. 2. ER versus average SNR for different values of the fading parameters.

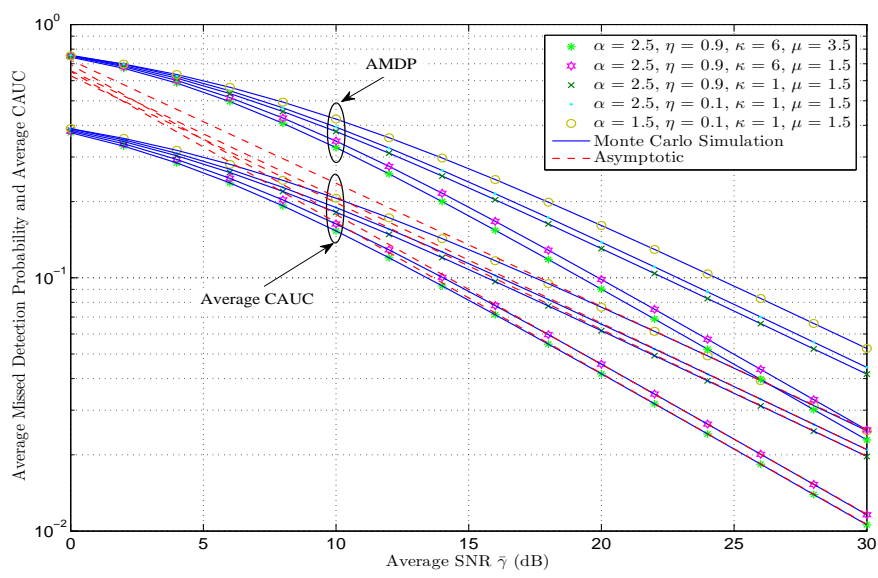

Fig. 3. Average probability of missed detection and average CAUC versus average SNR for different values of the fading parameters.

the total number of the signal levels which would allow to transmit more bits per second. Moreover, in both figures, it can be observed that the ER improves and both the $\bar{P}_{m d}$ and the average CAUC decrease when $\alpha, \eta, \kappa$, and/or $\mu$ increase. This is because the high value of $\alpha$ means the system tends to be linear whereas a large $\eta$ indicates that the total power of the in-phase components of the scattered waves is larger than that of quadrature counterparts. Additionally, the increasing in $\kappa$ makes the total power of the scattered waves is higher than that of the dominant components and high $\mu$ means a large number of multipath clusters arrive at the receiver. For example, in Fig. 2 , at $\bar{\gamma}=20 \mathrm{~dB}$ and $(\eta, \kappa, \mu)=(0.1,1,1.5)$, the ER for $\alpha=1.5$ is roughly 3.108 while it's value for $\alpha=2.5$ is nearly 3.315 . In the same context, when $\eta$ becomes 0.9 , the ER increases by approximately $2.62 \%$ at fixed $\alpha=2.5$. In addition, the increase in $\kappa$ from 1 to 6 enhances the ER by nearly $10.6 \%$ for constant $\alpha, \eta$, and $\mu$ at $2.5,0.9$, and 1.5 , 


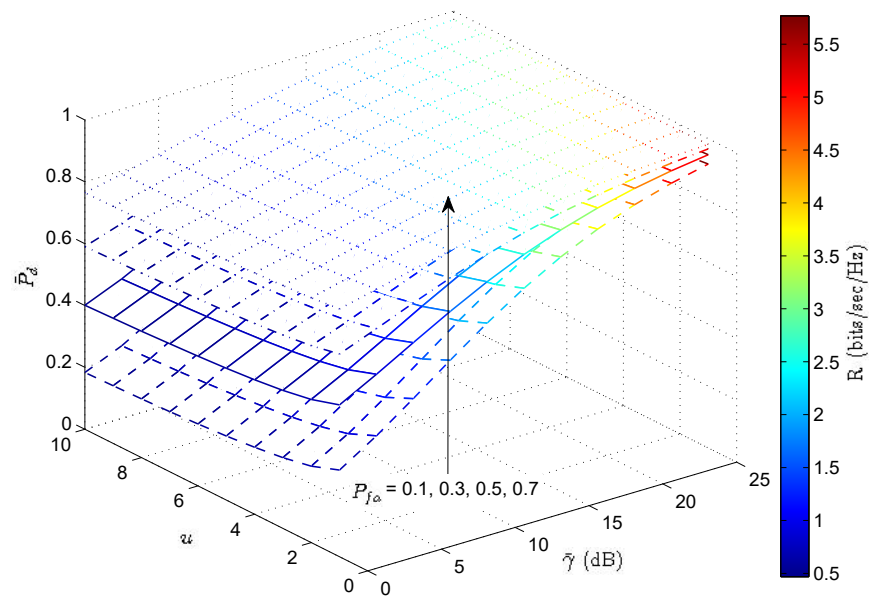

Fig. 4. ADP and ER versus average SNR and $u$ for different $P_{f a}$.

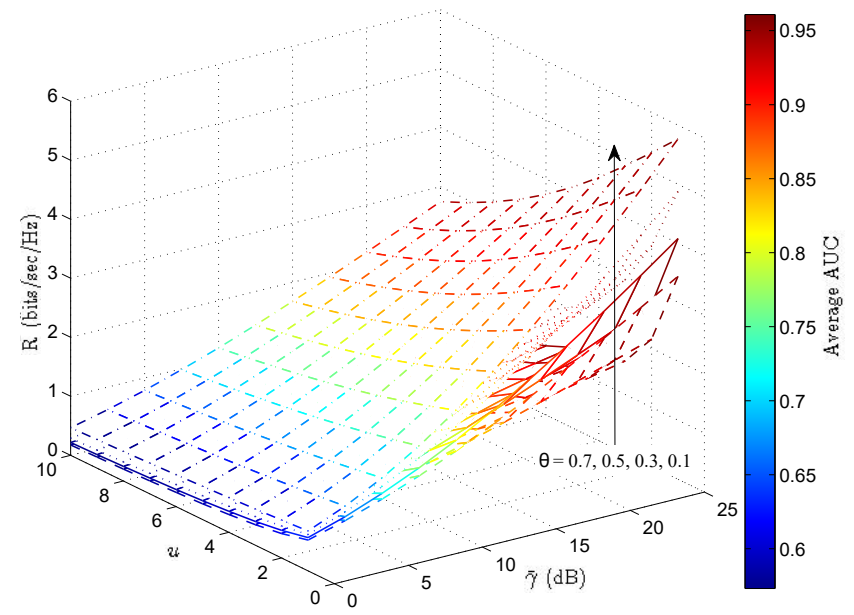

Fig. 5. ER and average AUC versus average SNR and $u$ for different $\theta$.

respectively. Similarly, when $(\alpha, \eta, \kappa)=(2.5,0.9,1)$, the ER for $\mu=1.5$ is 3.763 whereas it's value for $\mu=3.5$ is 3.894 .

In both Figs. 2 and 3, the exact results are perfectly matched with their asymptotic and Monte-Carlo simulation counterparts which proves the validation of our derived expressions.

To explain the relationship between the ER and ED, Fig. 4 demonstrates the ADP and ER versus $u$, and $\bar{\gamma}$, for $\alpha=1.5$, $\eta=0.1, \kappa=1, \mu=1.5, \theta=0.1$ and different values of $P_{f a}$. Additionally, Fig. 5 portraits the ER and average AUC versus $u$ and $\bar{\gamma}$. As expected, a substantial degradation in the ER and the detection capability of ED is noticed when $u$ increases and/or $\bar{\gamma}$ decreases. This is because the increasing in $u$ leads to reduce both the ADP and $P_{f a}$ simultaneously. However, the decreasing of the ADP is slower than the $P_{f a}$. In the same context, the average AUC becomes low because its computation depends on the area under the ROC curve. These observations are consistent with the results of [17] and [18]. Hence, the ER, i.e., number of the transmitted bits, reduces due to the increasing of the transmission delay and/or decreasing of the bandwidth as it can be seen in Figs. 4 and 5. Furthermore, the value of the ER rises with the decreasing of $\theta$ as a result of a large delay of the received signal. These situations are affirmed by the given results of [19]. Consequently, both the QoS and the performance of the ED are related and affected by $u$ as well as the fading parameters of the channel.

\section{CONCLUSions}

In this paper, the ER and the performance of ED based spectrum sensing over $\alpha-\eta-\kappa-\mu$ fading channels were analysed first using exact and asymptotic expressions. Thereafter, the mathematical relationship between the ER and ED was explained. This relationship is very important in providing good insights on how the ED of the signal and the QoS of the communication systems are affected by the time-bandwidth product. In addition, a series convergence acceleration for the PDF of $\alpha-\eta-\kappa-\mu$ fading was applied to find the number of the truncated terms. The numerical and simulation results for different scenarios were presented and showed that the system performance improves when $\alpha, \eta, \kappa$, and/or $\mu$ increase and $u$ decreases. The derived expressions can be used for various fading models, such as $\alpha-\kappa-\mu$ by plugging $\eta \rightarrow 1$.

\section{REFERENCES}

[1] M. K. Simon and M.-S. Alouini, Digital Communications over Fading Channels. New York: Wiley, 2005.

[2] M. D. Yacoub, "The $\kappa-\mu$ distribution and the $\eta-\mu$ distribution," IEEE Antennas Propag. Mag., vol. 49, no. 1, pp. 68-81, Feb. 2007.

[3] M. D. Yacoub, "The $\alpha-\mu$ distribution: a physical fading model for the Stacy distribution," IEEE Trans. Veh. Technol., vol. 56, no. 1, pp. 27-34, Jan. 2007.

[4] G. Fraidenraich and M. D. Yacoub, "The $\alpha-\eta-\mu$ and $\alpha-\kappa-\mu$ fading distributions," 2006, pp. 16-20, Manaus, Amazon, Brazil.

[5] M. D. Yacoub, "The $\alpha-\eta-\kappa-\mu$ fading model," IEEE Trans. Antennas Propag., vol. 64, no. 8, pp. 3597-3610, Aug. 2016.

[6] X. Li et al., "Capacity analysis of $\alpha-\eta-\kappa-\mu$ fading channels," IEEE Commun. Lett., vol. 21, no. 6, pp. 1449-1452, Jun. 2017.

[7] A. Mathur et al., "On physical layer security of $\alpha-\eta-\kappa-\mu$ fading channel," IEEE Commun. Lett., vol. 22, no. 10, pp. 2168-2171, Oct. 2018.

[8] J. M. Moualeu et al., "On the performance of $\alpha-\eta-\kappa-\mu$ fading channels," IEEE Commun. Lett., vol. 23, no. 6, pp. 967-970, June 2019.

[9] S. K. Yoo et al., "Entropy and energy detection-based spectrum sensing over $\mathcal{F}$ composite fading channels," IEEE Trans. Commun., vol. 67, no. 7, pp. 4641-4653, July 2019.

[10] I. S. Gradshteyn, and I. M. Ryzhik, Table of Integrals, Series and Products, 7th edition. Academic Press Inc., 2007.

[11] A. M. Mathai, R. K. Saxena, and H. J. Haubold, The H-Function: Theory and Applications. Springer, 2009.

[12] H. Al-Hmood and H. S. Al-Raweshidy, "Unified approaches based effective capacity analysis over composite $\alpha-\eta-\mu /$ gamma fading channels," Electronics Lett., vol. 54, no. 13, pp. 852-853, June 2018.

[13] F. F. Digham, M. S. Alouini, and M. K. Simon, "On the energy detection of unknown signals over fading channels," IEEE Trans. Commun., vol. 55, no. 1, pp. 21-24, Jan. 2007.

[14] H. Al-Hmood and H. S. Al-Raweshidy, (2019) "Selection combining scheme over non-identically distributed Fisher-Snedecor $\mathcal{F}$ fading channels," [Online]. Available: https://arxiv.org/pdf/1905.05595.pdf

[15] H. Chergui, M. Benjillali, and M.-S. Alouini, "Rician $K$-factor-based analysis of XLOS service probability in $5 \mathrm{G}$ outdoor ultra-dense networks, IEEE Wireless Commun. Lett., vol. 8, no. 2, pp. 428-431, Apr. 2018.

[16] W. Zeng, J. Zhang, S. Chen, K. P. Peppas, and B. Ai, "Physical layer security over fluctuating two-ray fading channels," IEEE Trans. Veh. Technol., vol. 67, no. 9, pp. 8949-8953, Sept. 2018.

[17] G. Chu, K. Niu, W. Wu and F. Yang, "MGF-based analysis of spectrum sensing over $\kappa-\mu$ fading channels for 5G cognitive networks," IEEE Access, vol. 6, pp. 78650-78658, Dec. 2018.

[18] S. Kumar, "Performance of ED based spectrum sensing over $\alpha-\eta-\mu$ fading channel," Wireless Personal Commun., vol. 100, no. 4, pp. 18451857, June 2018.

[19] S. K. Yoo et al., "A comprehensive analysis of the achievable channel capacity in $\mathcal{F}$ composite fading channels," IEEE Access, vol. 7, pp. 34078-34094, Feb. 2019. 